\title{
Systemtherapie des kolorektalen Karzinoms
}

Pestalozzi, B C ; Jäger, D ; Knuth, A

\begin{abstract}
Die medikamentöse Behandlung des kolorektalen Karzinoms hat in den letzten 10Jahren eindrückliche Fortschritte gemacht. Neben dem altbewährten 5-Fluorouracil stehen heute neue Zytostatika zur Verfügung wie Irinotecan und Oxaliplatin. Monoklonale Antikörper wie Bevacizumab und Cetuximab haben erfolgreich Eingang in aktuelle Therapiestrategien gefunden. Auf der Basis randomisierter klinischer Studien lassen sich heute rationale Therapiestrategien formulieren, wie in diesem Beitrag dargestellt
\end{abstract}

DOI: https://doi.org/10.1007/s00104-005-1038-6

Posted at the Zurich Open Repository and Archive, University of Zurich

ZORA URL: https://doi.org/10.5167/uzh-155715

Journal Article

Published Version

Originally published at:

Pestalozzi, B C; Jäger, D; Knuth, A (2005). Systemtherapie des kolorektalen Karzinoms. Der Chirurg, 76(6):570-574.

DOI: https://doi.org/10.1007/s00104-005-1038-6 
Chirurg 2005 $\cdot 76: 570-574$

DOI 10.1007/s00104-005-1038-6

Online publiziert: 19. Mai 2005

(c) Springer Medizin Verlag 2005
B. C. Pestalozzi · D. Jäger · A. Knuth

Klinik und Poliklinik für Onkologie, Universitätsspital Zürich, Schweiz er 1957 synthetisierte Antimetabolit 5Fluorouracil stellte jahrzehntelang das einzige Medikament dar, das routinemäßig in der Behandlung von Patienten mit kolorektalem Karzinom eingesetzt wurde. In klinischen Studien der 1970er und 1980er Jahre wurde die Wirksamkeit von 5-Fluorouracil durch die kontinuierliche Applikation und die biochemische Modulation mittels Leukovorin (Folinsäure) verbessert. Heute stehen auch orale Vorstufen des 5-Fluorouracils zur Verfügung, die als Monotherapie oder in Kombination eingesetzt werden. Erst in den 199oer Jahren haben sich die therapeutischen Möglichkeiten erheblich erweitert durch den Topoisomerase-IInhibitor Irinotecan und das Platinderivat Oxaliplatin in Kombination mit 5-Fluorouracil oder 5-Fluorouracil-Derivaten. Kürzlich wurde das therapeutische Spektrum in der Behandlung kolorektaler Karzinome wesentlich ergänzt durch die monoklonalen Antikörper Cetuximab und Bevacizumab, die an den EGFR (epithelial growth factor receptor) bzw. an den Liganden des VEGFR (vascular endothelial growth factor receptor) binden.

\section{Fluoropyrimidine}

5-Fluorouracil ist ein Antimetabolit, der die Zellproliferation hemmt durch Inhibition der Thymidilat-Synthetase, der DNSund der RNS-Synthese. Die Hemmung der Thymidilat-Synthetase, des einzigen metabolischen Wegs der Thymidinsynthese, kann durch Zugabe des Folsäurederivats Leukovorin verstärkt werden (bioche- mische Modulation). Viele Jahre wurde in klinischen Studien die optimale Applikationsform und Dosis des Fluorouracils und des Leukovorins gesucht. Einerseits hat man verschiedene Dosen von Leukovorin getestet. In randomisierten Studien fand sich kein Vorteil für das (kostspielige) „High-dose-Leukovorin“ (500 mg/ $\mathrm{m}^{2}$ ) gegenüber dem „Low-dose-Leukovorin“ $\left(20 \mathrm{mg} / \mathrm{m}^{2}\right)$ [10]. Andererseits wurden verschiedene Anwendungsarten von Fluorouracil studiert. Die Bolusapplikation, die kontinuierliche Applikation während 24 oder 48 h oder gar die niedrigdosierte 5 Fluorouracil-Gabe über viele Wochen. Bei den Bolusapplikationen wurden insbesondere zwei Schemen verwendet:

- das „Mayo-Schedule“ (täglich mit „Low-dose-Leukovorin“ während $5 \mathrm{Ta}$ gen jeden Monat) und

- das „Roswell-Park-Schedule“ (wöchentlich mit „High-dose-Leukovorin“).

Da 5-Fluorouracil eine kurze Halbwertszeit von wenigen Minuten hat, ist es nicht erstaunlich, dass die kontinuierliche Applikation der Bolusapplikation überlegen ist. Wegen unsicherer Resorption kann 5 Fluorouracil nicht oral verabreicht werden. Zur kontinuierlichen intravenösen Applikation ist in der Regel ein Port und ein Ballonpumpensystem nötig. Um dies zu umgehen, wurden verschiedene orale Präparate, u. a. Doxifluridine, Uracil-Tegafur, Ethynil-Uracil als so genannte "5Fluorouracil-Pro-Drugs“ (Vorstufen) ent- wickelt, mit denen die kontinuierliche Applikation imitiert werden kann. Heute steht in Deutschland, Österreich und der Schweiz nur Capecitabine (Xeloda ${ }^{\bowtie}$ ) als orale Vorstufe von 5-Fluorouracil zur Verfügung.

\section{( Mit sog. „Pro-Drugs" wird die kontinuierliche Applikation von 5-Fluorouracil imitiert}

Capecitabine ist in zwei großen randomisierten Studien gegenüber dem konventionellen Mayo-Regime mit Bolus-5-Fluorouracil getestet worden $[8,15]$. Dabei zeigte sich eine gewisse Verbesserung bezüglich der Ansprechrate (RR, „response rate“) und ein vergleichbares progressionsfreies Überleben (PFS, „progression free survival“) bzw. Gesamtüberleben (OS, „overall survival“). Die gastrointestinale und hämatologische Toxizität von Capecitabine war geringer als diejenige des Mayo-Regimes. Dafür trat häufiger ein meist mildes HandFuß-Syndrom auf.

Capecitabine ist geeignet für Patienten, bei denen eine Kombinationsbehandlung riskant erscheint und deren Zuverlässigkeit bei der Tabletteneinnahme gewährleistet ist. Capecitabine kann wie Fluorouracil mit Irinotecan bzw. Oxaliplatin kombiniert werden. Solche Kombinationen wurden in vielen Phase-II-Studien untersucht. Allerdings fehlen vergleichende Studien, welche erlauben würden, generell das kontinuierlich zu infundierende und mit Leukovorin ergänzte 5-Fluorouracil durch das orale Capecitabine zu ersetzen. 
Das Kolon- und die hohen Rektumkarzinome metastasieren in der Regel primär hämatogen in die Leber. Im Gegensatz zum Leberparenchym, dessen Blutversorgung hauptsächlich durch die Portalvenen erfolgt, werden Metastasen fast ausschließlich arteriell versorgt. Deshalb wird seit Jahren die lokoregionäre Chemotherapie über einen Katheter in der Arteria hepatica studiert. Vorzugsweise werden dabei Medikamente verwendet, die von der Leber beim ersten Durchgang weitgehend metabolisiert werden („first-pass effect“) und deshalb kaum systemische Nebenwirkungen verursachen, wie z. B. Fluoro-Uridine-Desoxy-Ribose (FUDR). Obwohl Lebermetastasen besser auf eine intraarterielle Therapie mit FUDR anzusprechen scheinen als auf intravenöses Fluorouracil, hat sich diese Therapieform nicht allgemein durchgesetzt und bleibt somit Forschungsgegenstand. Vielleicht bringt die Kombination der lokoregionären FUDRBehandlung mit den neu verfügbaren systemischen Medikamenten weitere Fortschritte.

\section{Irinotecan}

Irinotecan, CPT-11 (Campto ${ }^{\circledR}$ ), ist ein Hemmer der Topoisomerase I. Die Inhibition der Topoisomerasen führt zu Brüchen in der DNS. In einer langen Serie von klinischen Studien - Phase I (Dosisfindung), Phase II (Wirksamkeit), und Phase III (randomisierte Vergleichsstudie) - wurde die Wirksamkeit von Irinotecan in der Behandlung des kolorektalen Karzinoms nachgewiesen. Irinotecan verbessert das Überleben im Vergleich mit „,best supportive care“. Irinotecan ist wirksam auch bei Resistenz auf 5-Fluorouracil. Besser als die Monotherapie mit Irinotecan ist die Kombination mit 5-Fluorouracil und Leukovorin [12]. Auch in Kombination mit Irinotecan ist die kontinuierliche Gabe des 5-Fluorouracils der Bolusapplikation vorzuziehen [3, 11].

\section{(7) Besser als die Irinotecan- Monotherapie ist die Kombina- tion mit 5-Fluorouracil und Leukovorin}

In drei großen randomisierten Studien wurden 5-Fluorouracil und Leukovorin ge- gen dieselben Medikamente plus Irinotecan verglichen (• Tabelle 1) $[3,11,12]$. In allen drei Studien wurden die Remissionsraten, die progressionsfreie Überlebenszeit und das Überleben verbessert. In den USA wurde das IFL-Regime (Irinotecan plus Bolus-Fluorouracil und Leukovorin) zur Standardtherapie ab dem Jahre 2000 [12], während in Europa die kontinuierliche 5Fluorouracil-Applikation vorgezogen wurde $[3,11]$. Die deutsche Arbeitsgruppe für Internistische Onkologie (AIO) verwendet wöchentlich eine 24-stündige Infusion, während in Frankreich alle 2 Wochen eine 48-stündige Infusion zur Anwendung kommt (deGramont) [3].

Diese 5-Fluorouracil-Applikation mit 1- bis 2-tägiger Infusion scheint wirksamer und weniger toxisch $\mathrm{zu}$ sein als die Bolusapplikation. In etwa $20 \%$ der Irinotecan-behandelten Patienten kann es zu schweren Diarrhöen kommen. Bei gleichzeitig bestehender Neutropenie ist besonders bei älteren Patienten eine engmaschige Überwachung und frühzeitige Behandlung notwendig. Irinotecan soll nur von Spezialisten angewendet werden, die mit der Überwachung und den supportiven Maßnahmen (Loperamid, Antibiotika, Flüssigkeitsersatz) vertraut sind. Ferner kann Irinotecan auch zu Alopezie und einem so genannten „Fatigue-Syndrom “ führen neben anderen eher selteneren unerwünschten Wirkungen.

\section{Oxaliplatin}

Oxaliplatin (Eloxatine ${ }^{\bowtie}$ ) ist ein Platinanalog, das DNS-Addukte bildet. Oxaliplatin ist in der Monotherapie nur wenig wirksam beim kolorektalen Karzinom, es sollte deshalb immer mit einem Fluoropyrimidin kombiniert werden. Die Zugabe von Oxaliplatin zu 5-Fluorouracil und Leukovorin gegenüber diesen allein wurde in 3 großen Studien geprüft (• Tabelle 1$)[2,4$, $6]$. In allen Studien zeigte sich eine Verbesserung der Ansprechrate und des progressionsfreien Intervalls. Die Verlängerung der Überlebenszeit war nicht statistisch signifikant. Erst die große amerikanische Studie von Goldberg, welche die Überlegenheit von FOLFOX (Folinsäure = Leukovorin, 5-Fluorouracil, Oxaliplatin) gegenüber dem IFL-Regime ergab, führte dazu, dass FOLFOX heute auch in den USA
Chirurg 2005 $\cdot 76: 570-574$

DOI 10.1007/s00104-005-1038-6

○) Springer Medizin Verlag 2005

B. C. Pestalozzi · D. Jäger · A. Knuth

\section{Systemtherapie des kolorektalen Karzinoms}

\section{Zusammenfassung}

Die medikamentöse Behandlung des kolorektalen Karzinoms hat in den letzten 10 Jahren eindrückliche Fortschritte gemacht. Neben dem altbewährten 5-Fluorouracil stehen heute neue Zytostatika zur Verfügung wie Irinotecan und Oxaliplatin. Monoklonale Antikörper wie Bevacizumab und Cetuximab haben erfolgreich Eingang in aktuelle Therapiestrategien gefunden. Auf der Basis randomisierter klinischer Studien lassen sich heute rationale Therapiestrategien formulieren, wie in diesem Beitrag dargestellt.

\section{Schlüsselwörter}

Kolorektales Karzinom · Irinotecan .

Oxaliplatin - Capecitabine - Bevacizumab . Cetuximab

\section{Systemic therapy for colorectal cancer}

\section{Abstract}

Drug treatment of colorectal cancer has made impressive progress during the past 10 years. In addition to the traditional 5 -fluorouracil, newer anticancer drugs are available including irinotecan and oxaliplatin. Monoclonal antibodies like bevacizumab and cetuximab have been integrated into modern treatment regimens. Based on randomized clinical trials we can formulate rational treatment strategies as outlined in this article.

\section{Keywords}

Colorectal cancer · Irinotecan - Oxaliplatin . Capecitabine - Bevacizumab · Cetuximab 
Tabelle 1

\section{Erstlinientherapie in Phase-III-Studien}

\begin{tabular}{|c|c|c|c|c|}
\hline & $\mathbf{N}$ & $\begin{array}{l}\text { RR } \\
\text { [\%] }\end{array}$ & $\begin{array}{l}\text { TTP/PFS } \\
\text { [Mo] }\end{array}$ & $\begin{array}{l}\text { OS } \\
\text { [Mo] }\end{array}$ \\
\hline Capecitabine vs. & 301 & 19 & 5,2 & 13,2 \\
\hline • Mayo [10] & 301 & 15 & 4,7 & 12,1 \\
\hline Capecitabine vs. & 302 & 25 & 4,3 & 12,5 \\
\hline • Mayo [15] & 303 & 16 & 4,7 & 13,3 \\
\hline AIO/deGramont vs. & 199 & 23 & 4,4 & 14,1 \\
\hline - AIO/deGramont + Irinotecan [8] & 188 & 41 & 6,7 & 17,4 \\
\hline IFL (Saltz) vs. & 231 & 39 & 7,0 & 14,8 \\
\hline - Mayo vs. & 226 & 21 & 4,3 & 12,6 \\
\hline Irinotecan Monotherapie [3] & 226 & 18 & 4,2 & 12,0 \\
\hline AlO vs. & 216 & 32 & 6,4 & 16,9 \\
\hline - AIO + Irinotecan [12] & 214 & 54 & 8,5 & 20,1 \\
\hline deGramont vs. & 210 & 22 & 6,2 & 14,7 \\
\hline - FOLFOX [11] & 210 & 51 & 9,0 & 16,2 \\
\hline 5-FU + LV (chronomoduliert) vs. & 100 & 16 & 6,1 & 19,9 \\
\hline - FU + LV (chronomoduliert) + Oxaliplatin [2] & 100 & 53 & 8,7 & 19,4 \\
\hline Mayo vs. & 124 & 23 & 5,3 & 16,1 \\
\hline - FU+ LV + Oxaliplatin [4] & 114 & 49 & 7,8 & 21,4 \\
\hline IFL vs. & 264 & 31 & 6,9 & 15,0 \\
\hline - FOLFOX vs. & 267 & 45 & 8,7 & 19,5 \\
\hline Irinotecan + Oxaliplatin [6] & 264 & 35 & 6,5 & 17,4 \\
\hline FOLFIRI vs. & 109 & 56 & 8,5 & 20,2 \\
\hline - FOLFOX [5] & 111 & 54 & 8,1 & 21,5 \\
\hline aCetuximab vs. & 218 & 11 & 1,5 & ns \\
\hline 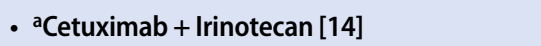 & 111 & 23 & 4,1 & ns \\
\hline IFL + Plazebo vs. & 412 & 35 & 6,2 & 15,6 \\
\hline - IFL + Bevacizumab [1] & 403 & 45 & 10,6 & 20,3 \\
\hline
\end{tabular}

N Anzahl Patienten pro Studienarm, RR response rate, TTP time to progression, PFS progression free survival, OS overall survival, AIO Arbeitsgruppe für Internistische Onkologie, IFL Irinotecan + Fluorouracil + Leukovorin, FOLFOX Folinsäure + 5-Fluorouracil + Oxaliplatin, FOLFIRI Folinsäure + 5-Fluorouracil + Irinotecan.

a Einzige Studie mit Vorbehandlung, in diesem Fall: Progression unter Irinotecan. als ein wichtiger Standard in der Behandlung des metastasierten kolorektalen Karzinoms gilt [5].

\section{จ Die Hauptnebenwirkung von Oxaliplatin ist die Neurotoxizität}

Hauptnebenwirkung von Oxaliplatin ist die Neurotoxizität. Eine reversible sensomotorische Neuropathie tritt in Abhängigkeit der kumulativen Dosis auf und wirkt dosislimitierend. Zudem muss der Patient auf eine meist selbstlimitierende akute kälteassoziierte Neuropathie aufmerksam gemacht werden, die sich als pharyngolaryngeale Dysästhesie oder als periphere Paräs- thesie manifestiert. Oxaliplatin ist nur mäBig hämatotoxisch und führt selten zu Alopezie.

\section{Oxaliplatin oder Irinotecan?}

Die Überlegenheit des Oxaliplatin-haltigen FOLFOX über das Irinotecan-haltige IFL-Regime [5] beweist keineswegs, dass Oxaliplatin dem Irinotecan überlegen ist. Sie könnte nämlich daran liegen, dass die Bolusapplikation des 5-Fluorouracils im IFL der kontinuierlichen Applikation im FOLFOX unterlegen ist. Für diese Interpretation spricht die Studie von Tournigand, welche auch Irinotecan mit dem kontinuierlich infundierten zu FOLFIRI kombi- niert [14]. In dieser Studie wurde die Sequenz FOLFIRI gefolgt von FOLFOX bei Progression verglichen mit der umgekehrten Sequenz, FOLFOX gefolgt von FOLFIRI. Im Gesamtüberleben ergab sich kein Unterschied zwischen diesen Sequenzen, die also als gleichwertig anzusehen sind. Das Zweitlinien-FOLFOX $(n=81)$ zeigte ein etwas besseres Ansprechen (14 vs. 4\%) und ein besseres progressionsfreies Überleben (4,1 vs. 2,5 Monate) als das Zweitlinien-FOLFIRI $(n=69)$. Die Antwort auf die Frage „Oxaliplatin oder Irinotecan?“ lautet also:

\section{"Beide, sequenziell nacheinander", wobei die optimale Reihenfolge noch nicht sicher geklärt ist.}

\section{Fazit zur Chemotherapie}

Auf der Basis der genannten Studien ( $\bullet$ Tabelle 1) ergeben sich heute folgende Schlüsse für die Behandlung des metastasierten kolorektalen Karzinoms [13]:

- Die so genannten „Doublets“ (FOLFOX oder FOLFIRI) sind die wirksamsten Kombinationen.

- Die Triplets (Oxaliplatin, Irinotecan, 5-Fluorouracil gleichzeitig) sind den Doublets therapeutisch nicht überlegen bei größerer Toxizität.

- 5-Fluorouracil ist weiterhin ein zentraler Bestandteil der wirksamsten Regimes.

- Kontinuierlich infundiertes 5-Fluorouracil ist sowohl als Monotherapie als auch in Kombination mit Irinotecan oder Oxaliplatin der Bolusgabe überlegen. Das monatliche Bolus-5-Fluorouracil-Regime (so genanntes Mayo-Regime) gilt heute als überholt.

- Mediane Überlebenszeiten von 20 Monaten und mehr können erreicht werden unter sequenziellem Einsatz der therapeutisch bedeutendsten drei Medikamente [7].

\section{Neue monoklonale Antikörper: Cetuximab und Bevacizumab}

Cetuximab ist ein humanisierter monoklonaler Antikörper gegen den Membranrezeptor „epithelial growth factor receptor" (EGFR). In einer Phase-III-Studie konnte 
Hier steht eine Anzeige.

型 Springer 
gezeigt werden, dass bei Irinotecan-resistenten Karzinomen die Kombination von Irinotecan mit Cetuximab ein nochmaliges Ansprechen ergeben kann (• Tabelle 1) [1]. Auch das progressionsfreie Überleben, nicht aber das Überleben, wurde davon beeinflusst. Cetuximab (Erbitux ${ }^{\circledast}$ ) ist in der Europäischen Union und in der Schweiz zugelassen, jedoch nur in Kombination mit Irinotecan und für die spezielle Situation der Irinotecan-resistenten Karzinome.

Im Jahre 2004 wurden beachtenswerte Resultate der Kombination von Bevacizumab mit IFL (Irinotecan, 5-Fluorouracil, Leukovorin) publiziert [9]. Bevacizumab ist ein monoklonaler humanisierter Antikörper gegen den Wachstumsfaktor der Angiogenese ,vascular endothelial growth factor" (VEGF). In einer großen randomisierten Studie von 815 Patienten mit fortgeschrittenem Kolorektalkarzinom war die Kombination von IFL mit Bevacizumab derjenigen mit Plazebo bezüglich Ansprechen, Zeit zur Progression und auch Überleben signifikant überlegen (• Tabelle 1) [9]. Nebenwirkungen von Bevacizumab sind arterielle Hypertonie und seltene gastrointestinale Blutungen. Bevacizumab $\left(\right.$ Avastin $\left.^{\circledast}\right)$ ist seit kurzem auch in der Europäischen Union und in der Schweiz erhältlich. Es ist zugelassen in Kombination mit intravenös verabreichtem 5-Fluorouracil/Leukovorin bzw. mit 5-Fluorouracil/ Leukorovin/Irinotecan als Erstlinientherapie des metastasierten kolorektalen Karzinoms.

Neben weiteren Antikörpern werden zurzeit auch andere Substanzen getestet beim kolorektalen Karzinom: Die Tyrosinkinasehemmer Gefitinib (ZD1839, Ires$\mathrm{sa}^{\oplus}$ ), Erlotinib (OSI-774, Tarceva ${ }^{\oplus}$ ) sowie der orale Angiogeneseinhibitor PTK 787 / ZK 222586. Auch neue Zytostatika wie Epothilone B und die Inhibitoren der Cyclo-Oxygenase II (Cox-II-Inhibitoren) werden studiert.

\section{Fazit für die Praxis}

Zusammenfassend ist festzuhalten, dass die Entwicklung der letzten 10 Jahre eine erfreuliche Erweiterung der therapeutischen Möglichkeiten in der Behandlung des kolorektalen Karzinoms gebracht hat mit stetig steigenden Überlebenswahrscheinlichkeiten.

\section{Korrespondierender Autor \\ Prof. Dr. A. Knuth}

Klinik und Poliklinik für Onkologie, Universtitätsspital Zürich, 8091 Zürich, Schweiz E-Mail: alexander.knuth@usz.ch

Interessenkonflikt: Keine Angaben

\section{Literatur}

1. Cunningham $D$, Humblet $Y$, Siena $D$, Khayat $D$ et al. (2004) Cetuximab monotherapy and cetuximab plus irinotecan in irinotecan-refractory metastatic colorectal cancer. N Engl J Med 351:337

2. De Gramont A, Figer A, Seymour M et al. (2000) Leucovorin and fluorouracil with or without oxaliplatin as first-line treatment in advanced colorectal cancer. J Clin Oncol 18:2938

3. Douillard JY, Cunningham D, Roth AD et al. (2000) Irinotecan combined with fluorouracil compared with fluorouracil alone as first-line treatment for metastatic colorectal cancer: A multicentre randomised trial. Lancet 355:1041

4. Giacchetti S, Perpoint B, Zidani R et al. (2000) Phase III multicenter randomized trial of oxaliplatin added to chronomodulated fluorouracil-leucovorin as first-line treatment of metastatic colorectal cancer. J Clin Oncol 18:136

5. Goldberg RM, Sargent DJ, Morton RF et al. (2004) A randomized controlled trial of fluorouracil plus leucovorin, irinotecan, and oxaliplatin combinations in patients with previously untreated metastatic colorectal cancer. J Clin Oncol 22:23

6. Grothey A, Deschler B, Kroening H et al. (2002) Phase Ill study of bolus 5-Fluorouracil (5-FU)/folinic acid (FA) (Mayo) vs weekly high-dose $24 \mathrm{~h} 5$-FU infusion/FA + oxaliplatin (OXA) (FUFOX) in advanced colorectal cancer (ACRC). Proc Am Soc Clin Oncol 21:129 (Abstr 512)

7. Grothey A, Sargent D, Goldberg RM, Schmoll HJ (2004) Survival of patients with advanced colorectal cancer improves with the availability of fluorouracil-leucovorin, irinotecan, and oxaliplatin in the course of treatment. J Clin Oncol 22:1209

8. Hoff PM, Ansari R, Batist G et al. (2001) Comparison of oral capecitabine versus intravenous fluorouracil plus leucovorin as first-line treatment in 605 patients with metastatic colorectal cancer: Results of a randomized phase III study. J Clin Oncol 19:2282

9. Hurwitz H, Fehrenbacher L, Novotny W, Cartwright et al. (2004) Bevacizumab plus irinotecan, fluorouracil, and leucovorin for metastatic colorectal cancer. N Engl J Med 350:2335

10. Jager E, Heike M, Bernhard H et al. (1996) Weekly high-dose leucovorin versus low-dose leucovorin combined with fluorouracil in advanced colorectal cancer: results of a randomized multicenter trial. Study Group for Palliative Treatment of Metastatic Colorectal Cancer Study Protocol 1. J Clin Oncol 14:2274

11. Koehne $\mathrm{CH}$, Van Cutsem E, Wils J et al. (2003) Irinotecan improves the activity of the AIO regimen in metastatic colorectal cancer: Results of EORTC GI Group study 40986. Proc Am Soc Clin Oncol 22:254 (Abstr 1018)
12. Saltz LB, Cox JV, Blanke C et al. (2000) Irinotecan plus fluorouracil and leucovorin for metastatic colorectal cancer. Inrinotecan Study Group. N Engl J Med 343:905

13. Sobrero AF (2004) Scheduling of fluorouracil: A forget-me-not in the jungle of doublets. J Clin Oncol 22:4

14. Tournigand C, Andre T, Achille E et al. (2004) FOLFIRI followed by FOLFOX6 or the reverse sequence in advanced colorectal cancer: A randomized GERCOR study. J Clin Oncol 22:229

15. Van Cutsem E, Twelves C, Cassidy J et al. (2001) Oral capecitabine compared with intravenous fluorouracil plus leucovorin in patients with metastatic colorectal cancer: Results of a large phase III study. J Clin Oncol 19:4097 